\title{
TTR
}

Traduction, terminologie, rédaction

\section{Le soutien accordé par les organismes subventionnaires pour les traductions anglaises de romans canadiens-français du $\mathrm{XIX}^{\mathrm{e}}$ siècle}

\section{Alexandra Hillinger}

Volume 32, numéro 1, 1er semestre 2019

Traduction et politique(s)

Translation, Politics and Policies

URI : https://id.erudit.org/iderudit/1068016ar

DOI : https://doi.org/10.7202/1068016ar

Aller au sommaire du numéro

Éditeur(s)

Association canadienne de traductologie

ISSN

0835-8443 (imprimé)

1708-2188 (numérique)

Découvrir la revue

Citer cet article

Hillinger, A. (2019). Le soutien accordé par les organismes subventionnaires pour les traductions anglaises de romans canadiens-français du XIX ${ }^{\mathrm{e}}$ siècle. TTR, 32(1), 113-143. https://doi.org/10.7202/1068016ar
Résumé de l'article

Dans cet article, nous examinons la production et la réception, dans l'Amérique du Nord anglophone, des traductions anglaises de trois romans canadiens-français publiés au XIX ${ }^{\mathrm{e}}$ siècle : L'Influence d'un livre (Aubert de Gaspé, fils, 1837), Les Anciens Canadiens (Aubert de Gaspé, père, 1863) et Angéline de Montbrun (Conan, 1884). Ces romans ont fait l'objet d'une première traduction (ou d'une retraduction) après l'adoption de la Loi sur les langues officielles en 1969 et la mise en place de subventions à la traduction du Conseil des arts du Canada. Ces événements ont contribué au renouvellement d'intérêt pour la traduction de la littérature canadienne-française, intérêt qui est également alimenté par un essor d'études et de publications dans le monde universitaire québécois. Dans cet article, nous décrivons le contexte de production des traductions Angéline de Montbrun (1974) et The Influence of a Book (1993) ainsi que de la retraduction Canadians of Old (1996). Nous nous attardons au financement de ces traductions, à savoir les subventions obtenues des organismes gouvernementaux et non gouvernementaux, notre objectif étant de montrer qu'en diminuant le risque financier encouru par les maisons d'édition, les différentes formes de soutien financier ont permis d'accroître le rayonnement de la littérature canadienne traduite. 


\title{
Le soutien accordé par les organismes subventionnaires pour les traductions anglaises de romans canadiens-français du XIX' ${ }^{\mathrm{e}}$ siècle
}

\author{
Alexandra Hillinger \\ Université Laval
}

\section{Résumé}

Dans cet article, nous examinons la production et la réception, dans l'Amérique $\mathrm{du}$ Nord anglophone, des traductions anglaises de trois romans canadiensfrançais publiés au XIX ${ }^{e}$ siècle : L'Influence d'un livre (Aubert de Gaspé, fils, 1837), Les Anciens Canadiens (Aubert de Gaspé, père, 1863) et Angéline de Montbrun (Conan, 1884). Ces romans ont fait l'objet d'une première traduction (ou d'une retraduction) après l'adoption de la Loi sur les langues officielles en 1969 et la mise en place de subventions à la traduction du Conseil des arts du Canada. Ces événements ont contribué au renouvellement d'intérêt pour la traduction de la littérature canadienne-française, intérêt qui est également alimenté par un essor d'études et de publications dans le monde universitaire québécois. Dans cet article, nous décrivons le contexte de production des traductions Angéline de Montbrun (1974) et The Influence of a Book (1993) ainsi que de la retraduction Canadians of Old (1996). Nous nous attardons au financement de ces traductions, à savoir les subventions obtenues des organismes gouvernementaux et non gouvernementaux, notre objectif étant de montrer qu'en diminuant le risque financier encouru par les maisons d'édition, les différentes formes de soutien financier ont permis d'accroittre le rayonnement de la littérature canadienne traduite.

Mots-clés : traduction de romans canadiens-français, L'Influence d'un livre, Les Anciens Canadiens, Angéline de Montbrun, appui à la traduction

\section{Abstract}

In this article, we look into the production and reception in anglophone North America of the English translations of three French-Canadian novels published in the $19^{\text {th }}$ century: L'Influence d'un livre (Aubert de Gaspé, fils, 1837), Les Anciens Canadiens (Aubert de Gaspé, père, 1863), and Angéline de Montbrun (Conan, 1884). These novels have been translated (or retranslated) since the introduction of the Official Languages Act in 1969 and the creation of 
the translation grants by the Canada Council for the Arts. These events, along with an increase in studies and publications by Québécois literature scholars, fuelled a renewed interest for the translation of French-Canadian literature. In this article, we will examine the production of the translations Angéline de Montbrun (1974) and The Influence of a Book (1993), as well as the retranslation Canadians of Old (1996), with a focus on their funding, i.e. the grants awarded by funding agencies. Our goal is to show that by reducing the financial risks for publishing houses, the different types of funding enabled an increase in visibility for translations of French-Canadian literature.

Keywords: translation of French-Canadian novels, The Influence of a Book, Canadians of Old, Angéline de Montbrun, support for translation

\section{Introduction}

De la Conquête jusqu'à la fin du XIX $\mathrm{X}^{\mathrm{e}}$ siècle, la littérature écrite dans le territoire que l'on désigne aujourd'hui comme le Québec s'emploie à servir la nation et s'adresse à un lecteur canadien-français (Biron et al., 2007, p. 57). En 1839, Lord Durham, qui perçoit les Canadiens français comme un peuple sans histoire et sans littérature, dépose son fameux rapport prônant leur assimilation (Lambton, 1839). À partir de ce moment, la production littéraire devient synonyme de survie. En ce sens, le XIX ${ }^{\mathrm{e}}$ siècle est caractérisé par le développement de la vie littéraire dans le territoire habité par les anciens colons français. On assiste à la naissance de journaux et de revues qui contribueront à la diffusion de la littérature canadienne-française, les plus marquants étant Le Foyer canadien (1863-1866), Les Soirées canadiennes (1861$1865)$ et Le Répertoire national (1848-1851) ${ }^{1}$. Les romans y paraitront sous forme de feuilletons (Linteau et al., 1979, p. 328). En 1860, l'abbé Casgrain parlera d'un «mouvement littéraire en Canada» et l'investira d'une mission : «elle [la littérature canadienne-française] sera essentiellement croyante et religieuse» (cité dans Biron et al., 2007, p. 58). Pour lui, la littérature doit se mettre au service des valeurs de la nation canadienne-française (Lemire, 1980, p.xxiii). Ainsi, on assiste à une réimpression des œuvres produites en Nouvelle-France afin de promouvoir le patriotisme canadien-français et le sentiment d'appartenance à la nation; par exemple, les Euvres de Samuel de Champlain sont rééditées sous le patronage de l'Université Laval en 1870.

Edmond Lareau, rappelons-le, a soutenu que le trait distinctif du roman canadien-français est son caractère essentiellement nationaliste (1874, p. 274), qui s'exprime notamment par la description et la

1. Pour une analyse approfondie des revues littéraires canadiennes-françaises et de leur contribution à la création d'un mouvement littéraire, voir Lemire (1994). 
glorification de la vie rurale et religieuse. Tel est le cas du roman Les Anciens Canadiens de Philippe Aubert de Gaspé, père (1863). Force est de constater que la production littéraire au Canada français dans la seconde moitié du XIX ${ }^{\mathrm{e}}$ siècle est assez modeste : on recense une soixantaine d'œuvres romanesques, une centaine de recueils en vers et environ 80 pièces de théâtre (Hayne, 1986, p. 56). Cette petite production s'explique par le fait que les textes de fiction sont considérés comme étant futiles, tant par les lecteurs que par les auteurs (Biron et al., 2007, p. 128).

On assiste par ailleurs à une accélération des activités de traduction à partir de 1850, particulièrement la traduction juridique et administrative (Delisle, 1987). À la suite de la Confédération canadienne en 1867, le français et l'anglais sont reconnus comme les langues officielles du Parlement canadien, des cours fédérales et de la province de Québec, ce qui augmente la demande de traduction pour les textes parlementaires et les textes de loi (Delisle, 2001). La traduction littéraire est à l'époque une activité des plus marginales. Dans son article «Literary Translation in Canada: A Survey» (1977), Philip Stratford affirme qu'avant 1920, seules douze œuvres ont été traduites au Canada (dix vers l'anglais et deux vers le français). À son avis, les deux groupes linguistiques se sont largement ignorés, laissant souvent aux grands centres littéraires que sont Paris, Londres et New York le soin de traduire la littérature canadienne (Stratford, 1977 , p. 37) ${ }^{2}$. Stratford note en outre qu'historiquement, la traduction au Canada s'est effectuée au Parlement et dans le secteur privé. Il postule donc que la grande quantité de textes à traduire produits par un pays officiellement bilingue fait en sorte que la traduction de textes pragmatiques domine au Canada. Il explique également que les traducteurs sont souvent des fonctionnaires anonymes, quoique compétents et bien rémunérés. Dans un tel contexte, la traduction littéraire était une activité marginale, perçue comme étant quelque peu futile et sans attrait économique (ibid., p. 38).

Il faudra attendre les années 1960 avant que la traduction littéraire prenne son envol au Canada. Selon Stratford, seulement une soixantaine d'œuvres littéraires ont été traduites avant cette période (ibid., p. 37). Soulignons que la mise sur pied du programme d'aide à la traduction par le Conseil des arts du Canada en 1972 a permis une

2. Il importe de nuancer les propos de Stratford. Puisque le développement de l'édition est assez lent tant au Canada français qu'au Canada anglais au XIX ${ }^{\mathrm{e}}$ siècle, une grande partie de l'édition littéraire a lieu dans les grands centres, en Europe et aux États-Unis. 
meilleure diffusion de la littérature par l'entremise de la traduction. Notons également la création du prix du Gouverneur général du Canada pour la traduction en 1974 et la constitution en 1975 de l'Association des traducteurs et traductrices littéraires du Canada, qui décerne depuis 1982 le prix John-Glassco visant à souligner l'excellence d'une première traduction littéraire.

Dans cet article, nous nous penchons sur le contexte de production et la réception des traductions anglaises de trois romans canadiens-français du XIX ${ }^{\mathrm{e}}$ siècle : L'Influence d'un livre (Aubert de Gaspé, fils, 1837), Les Anciens Canadiens (Aubert de Gaspé, père, 1863) et Angéline de Montbrun (Conan, 1884). Les traductions de ces romans paraissent à partir des années 1970 : Angéline de Montbrun fait l'objet d'une traduction en 1974, suivie de L'Influence d'un livre en 1993 et des Anciens Canadiens en 199633. Nous avons sélectionné ces œuvres parce qu'elles ont marqué, chacune à leur manière, la production littéraire de langue française au Canada. L'Influence d'un livre est considéré comme le premier roman issu du Canada français ${ }^{4}$, alors que Les Anciens Canadiens constitue le premier succès commercial littéraire du $\mathrm{XIX}^{\mathrm{e}}$ siècle ${ }^{5}$. Quant à Angéline de Montbrun, il s'agit du premier roman psychologique, sans compter qu'il a été rédigé par la première femme à vivre de sa plume et à s'imposer sur la scène littéraire canadienne-française (Brunet, 2005, paragr. 6). Le facteur clé qui a influencé le choix des ouvres examinées dans notre étude est donc l'importance historique ${ }^{6}$ de celles-ci.

Dans le cadre de notre recherche, nous avons envisagé l'histoire de la pratique de la traduction en tant que processus et résultat. Selon

3. Il s'agit dans ce cas d'une nouvelle traduction, puisque le roman avait fait l'objet d'une première traduction en 1864 et d'une deuxième en 1890.

4. Voir à ce propos Hayne et Tirol (1968a) ainsi que Grutman et La Charité (2013). Dans la Bibliographie critique du roman canadien-français, Hayne et Tirol définissent le roman canadien-français comme «une narration fictive en prose, assez considérable pour occuper seule en un volume d'une centaine de pages, et ayant paru au moins une fois en volume séparé; rédigée en français pour des lecteurs adultes par un auteur né et élevé, ou résident de façon permanente, au Canada " (1968c, avant-propos, paragr. 3).

5. Hayne et Tirol écrivent au sujet des Anciens Canadiens: «Tiré à 1,100 exemplaires [...]. Le succès fut immédiat; il dure encore» (1968b, n. p.). De fait, la première édition s'est écoulée en quelques mois, et il en fut de même pour la suivante. C'est la raison pour laquelle Rainier Grutman et Claude La Charité estiment qu'Aubert de Gaspé, père, est «l'auteur du plus grand best-seller de l'époque» (2013, p. 1).

6. Nous n'examinons pas les deux premières traductions des Anciens Canadiens ni celle du roman À l'cuvre et à l'épreuve de Laure Conan, car elles ont été publiées avant 1920 et n'ont donc pas profité de financement extérieur. Nous traitons de ces romans dans notre thèse de doctorat (Hillinger, 2017). 
Judith Woodsworth, l'histoire de la pratique permet de répondre aux questions suivantes : qu'est-ce qui a été traduit, par qui, dans quelles circonstances et dans quel contexte sociopolitique (2001, p.101)? Il s'agit exactement des questions auxquelles nous avons tenté de répondre en analysant le contexte de production et de réception des traductions de L'Influence d’un livre, Les Anciens Canadiens et Angéline de Montbrun. Nous nous pencherons donc sur la nature de chacun de ces romans avant de nous attarder au traducteur et au contexte de production de la traduction, plus particulièrement au financement des projets de traduction. Nous examinerons également la réception critique de la traduction en Amérique du Nord.

On l'a dit, la production littéraire débute lentement au Canada français au XIX ${ }^{\mathrm{e}}$ siècle, et la diffusion de traductions anglaises dans l'Amérique du Nord anglophone est limitée. Puisque les francophones et les anglophones du Canada forment deux sociétés distinctes et que les échanges entre eux sont peu nombreux, il n'est pas surprenant qu'il n'y ait pas de désir de traduction au-delà d'entreprises ponctuelles. Cependant, malgré leur petit nombre, nous croyons que les traductions anglaises de romans canadiens-français ont permis une meilleure compréhension de l'histoire et de la culture canadiennes-françaises par les lecteurs anglophones - ce désir de compréhension étant au cœur des projets de traduction de la production romanesque du XIX ${ }^{\mathrm{e}}$ siècle. Nous remarquons également que la majorité des traductions produites à l'époque sont le résultat d'initiatives personnelles, à savoir celles de l'auteur des Anciens Canadiens, Aubert de Gaspé; du professeur et traducteur Yves Brunelle, grand défenseur de la littérature québécoise, de l'éditeur spécialisé dans les ouvrages québécois anciens, Robert Davies, et de la traductrice littéraire Jane Brierley, admiratrice des œuvres d'Aubert de Gaspé. Nous avons également constaté que ces acteurs ont porté à bout de bras leur projet de traduction des premiers romans canadiens-français, rendant accessibles aux lecteurs anglophones ces œuvres souvent méconnues. Ainsi, les versions an-

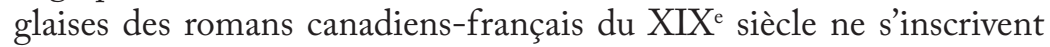
pas dans une tradition de traduction, c'est-à-dire qu'il n'y a jamais eu d'élan de traduction des œuvres du XIX ${ }^{\mathrm{e}}$ siècle et aucun effort institutionnel dans ce sens.

Dans cet article, nous nous pencherons sur la question du financement accordé par des organismes gouvernementaux et non gouvernementaux pour les traductions Angéline de Montbrun et The Influence of a Book ainsi que pour la retraduction Canadians of Old. Ce financement prend différentes formes et n'a pas toujours comme 
finalité la rémunération du traducteur. Malgré tout, nous postulons qu'il a permis de diminuer le risque financier encouru par les éditeurs, contribuant ainsi à la diffusion de la littérature canadienne-française à l'extérieur de ses frontières linguistiques.

\section{Angéline de Montbrun : l'ouvre originale ${ }^{7}$}

Angéline de Montbrun est le premier roman de Laure Conan. À la suite du décès de ses parents, la nécessité financière pousse Conan à se tourner vers l'écriture et à faire publier ses œuvres. De 1881 à 1882, Angéline de Montbrun paraît en feuilletons dans la Revue canadienne. Désireuse de publier son roman sous forme de livre, la romancière obtient le soutien de l'abbé Casgrain. Ce dernier, connu comme le "père» de la littérature canadienne-française ${ }^{8}$, met tout en œuvre pour aider la jeune romancière et la faire connaître. Il lui trouve un éditeur et demande entre autres à Louis Fréchette d'écrire une critique du roman (Brunet, 2005).

Laure Conan joue un rôle de précurseure à plusieurs égards. Selon Manon Brunet, elle est à l'époque la seule femme à vivre du métier d'écrivaine au Canada français (ibid.). Cependant, il est important de comprendre que ses écrits ne sont pas de nature féministe. La piété, le renoncement et le sacrifice de soi sont les thèmes le plus souvent associés aux personnages féminins qu'elle met en scène. Il n'en demeure pas moins que la romancière est consciente des barrières qui sont imposées aux femmes par la société québécoise patriarcale. C'est ce que révèlent notamment des lettres échangées avec le premier ministre du Canada, Sir Wilfrid Laurier. Le 6 avril 1907, elle lui écrit: «Si j'étais homme, on me traiterait bien autrement», et le 18 avril : «Tout va à ces Messieurs et je serai toujours sacrifiée si vous ne m'honorez de votre protection» (Conan cité dans Brunet, 2005). Angéline de Montbrun est en outre le premier roman psychologique de la littérature québécoise (Biron et al., 2007, p. 144). Contrairement au

7. La présentation est basée sur l'ordre chronologique de parution des traductions.

8. Cette image de l'abbé Casgrain prend racine à la fin du XIX siècle. Il faut savoir que Casgrain, sous le pseudonyme de Placide Lépine, se qualifie lui-même de «Père de l'Église littéraire» (Brunet, 1997, p. 219). L'universitaire Camille Roy sera le premier à évaluer de manière critique le travail de Casgrain, et il n'utilisera pas l'appellation «père». (ibid., p. 210) Manon Brunet explique que cette appellation n'est plus utilisée dans les manuels d'histoire littéraire depuis le début du $\mathrm{XX}^{\mathrm{e}}$ siècle (ibid., p. 206-207). Au final, ce sont principalement les contemporains de Casgrain (les auteurs canadiens-français du XIX ${ }^{\mathrm{e}}$ ) qui ont façonné cette image, dont Laure Conan et Napoléon Legendre (ibid., p. 212-214). 
roman de la terre ${ }^{9}$, le thème principal du livre de Conan est l'amour, et les sentiments sont à l'avant-plan.

\subsection{La traduction : Angéline de Montbrun}

La traduction anglaise d'Angéline de Montbrun paraît en 1974; elle est signée par Yves Brunelle, professeur au Département d'anglais de la St. Francis Xavier University, en Nouvelle-Écosse. Il ne s'agit pas de sa seule traduction, puisqu'il a aussi traduit Un homme et son péché de Claude Henri Grignon en 1978. Yves Brunelle a par ailleurs édité l'anthologie French Canadian Prose Masters: The Nineteenth Century (1978) et agi comme éditeur adjoint de la collection «French Writers of Canada", dont la mission est d'offrir aux lecteurs anglophones un échantillon des meilleures œuvres issues du Canada français (Grignon, $1978,2^{\text {e }}$ de couverture). Brunelle a beaucoup œuvré à la valorisation de la recherche sur la littérature canadienne et l'enseignement de celle-ci (Milner, 1997, p. 13). Tout porte à croire que c'est son intérêt pour la diffusion de la littérature canadienne et québécoise qui l'a motivé à entreprendre la traduction d'Angéline de Montbrun.

\subsection{Le projet de traduction}

Ce n'est qu'en 1974, près d'un siècle après sa publication en français, qu'Angéline de Montbrun fait l'objet d'une traduction vers l'anglais, un fait surprenant étant donné la bonne réception de l'œuvre à sa parution et les nombreuses études auxquelles elle a donné lieu au fil des ans. La traduction contient une introduction substantielle rédigée par le traducteur, qui y décrit le contexte de publication de l'œuvre et présente une biographie de l'auteure. Brunelle n'explique pas pourquoi il a entrepris la traduction de ce roman et ne fait pas non plus de commentaires sur le processus de traduction. Comme il est avant tout un professeur qui s'est donné comme mission de contribuer à la diffusion des œuvres québécoises, il dédie son introduction à la présentation et à la contextualisation du roman et de son auteure pour le public canadien-anglais peu familier avec les débuts de la littérature canadienne-française. La traduction est publiée dans la collection «Literature of Canada - Poetry and Prose in Reprint» des Presses de

9. «Roman de la terre» ou « roman du terroir» est l'appellation donnée à l'une des trois catégories majeures de la production romanesque québécoise jusqu'aux années 1930 ou 1940, les deux autres étant le roman historique et le roman d'aventures. Le roman du terroir décrit la réalité rurale où la campagne est perçue comme un endroit idéal procurant bonheur et prospérité. La campagne y est souvent mise en opposition avec la ville, qui représente un lieu de vices et de péchés (Boivin, 2006, p. 32). 
l'Université de Toronto. Selon son directeur Douglas Lochhead, cette collection a pour but de réimprimer des ouvrages de prose et de poésie afin de démontrer l'existence d'une littérature canadienne et d'en faire connaître les auteurs et les œuvres (Conan, 1974, n. p.). Une note contenue sur la page de renseignements relatifs à l'édition indique que l'ouvrage a bénéficié du soutien financier de deux organismes canadiens, à savoir le Conseil des arts de l'Ontario et la fondation McLean. La note se lit comme suit: «This book has been published with the assistance of grants from the Ontario Arts Council and the McLean Foundation»(ibid., n. p.).

Un échange de courriels avec Ev McTaggart, de la fondation McLean, nous a appris que les demandes de financement soumises à cet organisme et la correspondance s'y rattachant ne sont conservées que pendant deux ans. L'information relative au financement de la traduction d'Angéline de Montbrun a donc été détruite il y a près de quarante ans (Ev McTaggart, correspondance personnelle, 25 mai 2015). Du côté du Conseil des arts de l'Ontario, une correspondance avec Jack Illingworth, le responsable de la littérature, nous a appris que les archives de cette époque n’ont pas été conservées audelà des rapports annuels, que nous n'avons pu obtenir étant donné que les informations relatives aux subventions sont confidentielles. Jack Illingworth nous a toutefois indiqué que le Conseil des arts de l'Ontario accorde un soutien financier aux éditeurs ontariens depuis 1973. De plus, bien que les subventions aient été calculées titre par titre, les maisons d'édition indiquaient dans leur formulaire de demande toutes les publications prévues pour l'année en question. Ainsi, les Presses de l'Université de Toronto ont reçu une subvention de 15000 \$ en 1974, montant correspondant à la totalité du soutien financier offert par le Conseil cette année-là. Cette subvention a soutenu la publication de plusieurs titres, dont peut-être la traduction d'Angéline de Montbrun. Jack Illingworth nous a toutefois mise en garde contre le fait de supposer qu'une partie de ce montant a mené à la publication de la traduction d'Angéline de Montbrun. En effet, contrairement aux subventions à la traduction du Conseil des arts du Canada, qui sont accordées pour un livre précis, les subventions du Conseil des arts de l'Ontario qui ont été consenties aux Presses de l'Université de Toronto dans les années 1970 avaient pour but de réduire le risque financier encouru et de les aider à maintenir leur catalogue de publications (Jack Illingworth, correspondance personnelle, 5 août 2015). Par ailleurs, Charley LaRose, coordonnateur des publications des Presses 
de l'Université de Toronto, nous a indiqué que les archives de la maison d'édition sont conservées dans les archives de l'Université de Toronto. Cependant, comme les Presses n'ont pas reçu de financement précisément pour la publication de la traduction d'Angéline de Montbrun, on ne trouvera pas dans ces archives de correspondance à ce sujet (Charley LaRose, correspondance personnelle, 19 août 2015). Dans le cas de cette traduction, il est donc impossible de déterminer précisément comment les sommes allouées par la fondation McLean et le Conseil des arts de l'Ontario ont été utilisées. Considérant la note citée plus haut, nous nous permettons de conclure que ces organismes ont versé des montants à la maison d'édition afin de soutenir la publication (et non de rémunérer le traducteur). En effet, rien n'indique qu'Yves Brunelle a été rémunéré pour son travail.

\subsection{Réception de la traduction dans le Canada anglophone}

La traduction d'Yves Brunelle n'a pas fait l'objet d'étude critique, ce que constate David M. Hayne : «[i]1 est à regretter [...] qu'aucun traducteur ou traductologue n'ait examiné à fond la traduction anglaise du roman publiée en 1974 par Yves Brunelle» (2009, p. 620). Comme Angéline de Montbrun est un roman très étudié, la traduction de Brunelle est souvent mentionnée dans des articles et ouvrages parus depuis 1980, mais elle n'est jamais commentée comme telle, l'accent étant mis sur le roman original. Néanmoins, l'introduction écrite par Brunelle a été remarquée. Elle est mentionnée, entre autres, dans $A$ Critical Bibliography of French Literature (Brooks, 1980, p. 130), «The Québécois- and English-Canadian Literary System: Translation, Republishing, and the Preface» (Perkes, 1997, p.316-317) et "Consolation and the Work of Mourning in Angéline de Montbrun" (Carr, 1998, p. 1004). Carolyn Perkes, par exemple, explique que selon Brunelle, Angéline serait le premier « round character ${ }^{10}$ de la littérature québécoise (ibid., p. 48).

\section{L'Influence d'un livre : l'œuvre originale}

Publié en 1837 par Philippe-Ignace-François Aubert de Gaspé, journaliste et fils aîné du romancier Philippe Aubert de Gaspé, L'Influence

10. Un «flat character» est construit autour d'une seule idée ou d'une seule qualité; il est habituellement présenté en une phrase ou deux, avec très peu de détails. Un « round character» est un personnage complexe tant au niveau de son tempérament que de ses motivations; ce personnage est tout en subtilité et a le potentiel de surprendre le lecteur (Abrams et Harpham, 2015, p. 48). La distinction a été proposée par E.M. Foster dans son ouvrage Aspects of the Novel (1927). 
d'un livre est considéré comme le premier roman canadien-français ${ }^{11}$. De Gaspé ${ }^{12}$ est né à Québec le 8 avril 1814 et est décédé à Halifax le 7 mars 1841, à l'âge de 26 ans. Décrit comme un «turbulent contestataire» (Lacourcière, n. d., n. p.), de Gaspé eut une courte vie, mais elle fut mouvementée si l'on en juge par un événement survenu le 12 février 1836. Ce jour-là, le jeune journaliste, souhaitant se venger d'un député qui l'avait accusé d'avoir malhonnêtement rapporté les débats, jette de l'assa-foetida sur le poêle de la Chambre d'assemblée, ce qui répand une odeur nauséabonde dans tout l'édifice. LouisJoseph Papineau, alors orateur de la Chambre, lance un mandat d'arrestation contre de Gaspé à la suite de l'événement. Le jeune homme quitte Québec pour se réfugier au manoir seigneurial de Saint-JeanPort-Joli (Noreau, 2010, p. 61-63), propriété de sa famille. C'est là qu'il écrira L'Influence d'un livre.

L'œuvre a bénéficié d'une bonne réception, et ce, avant même sa publication, puisque la maison William Cowan et Fils a accepté de l'imprimer. Willam Cowan était un libraire-imprimeur d'envergure tenant boutique sur la rue de la Fabrique, à Québec. Avant 1840, les libraires anglophones imprimaient des ouvrages tant en français qu'en anglais (Galarneau, 1986, p. 4). Bien que L'Influence d'un livre ait été remarqué au moment de sa publication, les avis exprimés à son égard nétaient pas tous favorables. Il a notamment été qualifié d'invraisemblable par un journaliste utilisant le nom de plume "Pierre-André", ce qui a donné lieu à un échange entre ce dernier et Aubert de Gaspé dans La Gazette de Québec et Le Populaire (Hayne et Tirol, 1968a).

Le roman est réédité en 1864 par l'abbé Casgrain, qui en prépare aussi une version censurée pour le Foyer canadien. Casgrain a d'ailleurs changé le titre du roman pour Le chercheur de trésors, et c'est surtout sous ce titre que l'ouvrage a été connu.

\subsection{La traduction : The Influence of a Book}

Malgré les rééditions, le premier roman produit au Québec ne sera traduit vers l'anglais qu'un siècle et demi après sa parution originale. La traduction est signée par Claire Holden Rothman, romancière

11. L'auteur a fait l'objet d'une seule biographie, signée par Michel A. Noreau en 2010. La distribution de cette biographie autoéditée est assurée par le Musée de la mémoire vivante de Saint-Jean-Port-Joli, mis sur pied par la Corporation Philippe-Aubert-deGaspé sur les terres du domaine seigneurial de la famille Aubert de Gaspé.

12. Selon Noreau, Aubert de Gaspé fils utilisait simplement le patronyme «De Gaspé» (2010, p. XI-XIII). 
et traductrice ayant enseigné l'anglais au Collège Marianopolis de Montréal et la création littéraire à l'Université McGill (Open Book Toronto, 2009). Holden Rothman n'accompagne sa traduction d'aucune réflexion sur son travail. Selon ses souvenirs, c'est l'éditeur Robert Davies qui l'aurait approchée pour lui proposer de traduire le roman. Elle dit avoir été très enthousiasmée par le projet et heureuse d'être sollicitée pour traduire une œuvre littéraire de qualité (Claire Holden Rothman, communication personnelle, 31 mars 2016). Soulignons que Claire Holden Rothman a reçu en 1994 le prix JohnGlassco pour sa traduction, intitulée The Influence of a Book.

\subsection{Le projet de traduction}

La traduction de L'Influence d'un livre est publiée en 1993 chez Robert Davies Publishing, une division de l'Étincelle éditeur, et est distribuée au Canada par Stewart House. La maison d'édition a reçu le soutien du Conseil des arts du Canada ${ }^{13}$ pour la réalisation de la traduction et la publication de l'ouvrage, comme on peut le lire dans les pages liminaires de celui-ci : "The publisher gratefully acknowledges the support of the Canada Council for the translation and publication of this book» (De Gaspé, 1993, n.p.). Cependant, les informations relatives à cette subvention obtenue dans le cadre du programme "Aide à l'édition de livres : subventions à la traduction» ne sont plus disponibles. Debbie Stenson, coordonnatrice de l'accès à l'information et de la protection des renseignements personnels au Conseil des arts, nous a en effet informée que les documents relatifs aux subventions octroyées par le Conseil sont détruits après vingt ans (Debbie Stenson, communication personnelle, 30 mars 2016). C'est donc sur la base de la note mentionnée ci-dessus que nous en venons à la conclusion que l'éditeur a bénéficié d'une subvention spécifiquement destinée à rémunérer la traductrice, en plus d'une subvention de fonctionnement.

Une note d'un quart de page est la seule justification de l'éditeur quant à la production et à la publication, plus de 150 ans après sa parution originale, de cette traduction du premier roman canadienfrançais :

13. Rappelons que le Conseil des arts du Canada est un organisme public qui soutient «l'excellence artistique en offrant aux artistes et aux organismes artistiques canadiens une vaste gamme de subventions, de services, de prix et de paiements et [il en fait] la promotion» (Conseil des arts du Canada, n. d., n. p.). Dans le cadre de cette mission, il finance des activités liées à la traduction d'œuvres littéraires ou d'œuvres dramatiques en français, en anglais ou dans une langue autochtone [...] destinées à une publication ou à une présentation au pays (ibid.). 
This first Quebec Novel, published in the autumn of 1837, was penned by the son and namesake of the celebrated nineteenth-century author of Canadians of Old. The younger De Gaspé was a colourful personality, faithfully reflecting hopes and the effervescence of his time. In publishing this translation of one of the rare jewels of Canadian literature, it is our hopes that we have remained true to the feeling of that period and true to the intent of the author. (De Gaspé, 1993, n. p.)

L'entretien téléphonique que nous avons eu avec la traductrice nous a permis d'en apprendre un peu plus sur le contexte ayant donné lieu à la traduction. Selon Claire Holden Rothman, le fait que L'Influence d'un livre n'avait jamais été traduit vers l'anglais indignait Robert Davies. Ayant obtenu le nom d'Holden Rothman par l'intermédiaire de l'Association des traducteurs et traductrices littéraires du Canada, Davies lui aurait alors proposé de traduire le roman (Claire Holden Rothman, communication personnelle, 31 mars 2016). Dans sa critique de la traduction parue le 2 avril 1994 dans The Gazette, Wendy Thatcher écrit pour sa part que Davies s'était donné comme mission de faire connaître les textes québécois oubliés de la période allant de la fin du XVIII ${ }^{\mathrm{e}}$ siècle au début du XIX ${ }^{\mathrm{e}}$ siècle (Thatcher, 1994, p. I2). On peut penser que le projet de traduire L'Influence d'un livre s'inscrivait dans cette mission.

\subsection{Réception de la traduction dans le Canada anglophone}

L'éditeur choisit d'accompagner la version anglaise de L'Influence d'un livre d'une introduction signée par Maurice Lemire, professeur retraité de l'Université Laval, historien de la littérature et instigateur du Dictionnaire des cuvres littéraires du Québec et de L'Histoire de la vie littéraire du Québec. Si l'introduction fournit des informations biographiques sur l'auteur, résume la trame narrative et fait état des conditions de publication et de réception de l'original, elle ne contient par contre aucune mention de la traduction. En fait, l'introduction n'a pas été écrite spécialement pour l'occasion; il s'agit plutôt d'une traduction de l'article "L'Influence d'un livre, roman de PhilippeIgnace-François Aubert de Gaspé» paru en 1978 dans le premier tome du Dictionnaire des cuvres littéraires du Québec. Il est probable qu'on ait repris cet article parce que le nom de Maurice Lemire pouvait contribuer à conférer du prestige, de la crédibilité et une visibilité accrue à l'ouvrage. L'objectif de l'éditeur semble en outre avoir été de créer l'illusion d'une introduction écrite par Lemire spécialement pour l'occasion. En effet, l'introduction est simplement 
signée «Maurice Lemire» et n'est pas suivie de la date de la première publication du texte, à savoir 1978. De plus, l'article du Dictionnaire des ceuvres littéraires du Québec n'est pas cité dans la bibliographie commentée qui se trouve à la fin de l'ouvrage. Nous supposons donc qu'il y avait clairement un désir de présenter ce texte comme ayant été rédigé précisément pour accompagner cette première traduction anglaise de L'Influence d'un livre.

En ce qui a trait à la réception de la traduction, nous avons trouvé une annonce de sa parution et deux annonces relatives à l'obtention du prix John-Glassco par la traductrice. Nous avons également trouvé deux articles critiques, le premier déjà cité, publié dans le quotidien The Gazette du 2 avril 1994, et le deuxième, dans la revue Matrix (Cimon, 1994). Les deux articles mentionnent brièvement le travail de la traductrice. Dans The Gazette, on souligne qu'Holden Rothman reproduit avec justesse l'anglais du XIX ${ }^{\mathrm{e}}$ siècle (Thatcher, 1994, p. I2), alors que la critique de la revue Matrix met de l'avant le fait que le roman a été traduit avec adresse (Cimon, 1994, p. 76). Il est encore trop tôt pour déterminer si The Influence of a Book saura résister au passage du temps. Toutefois, il est difficile de faire des prédictions positives, car l'ouvrage n'a pas été réimprimé. De plus, selon Worldcat, seules 57 bibliothèques dans le monde en possèdent un exemplaire.

\section{Les Anciens Canadiens: l'ouvre originale}

Passons maintenant à la dernière retraduction des Anciens Canadiens de Philippe Aubert de Gaspé, père. Nous l'avons dit: Les Anciens Canadiens est considéré comme le premier grand succès commercial - et le plus grand - du XIX ${ }^{\mathrm{e}}$ siècle. L'ouvrage est un best-seller : selon l'abbé Casgrain, la première édition fut écoulée en quelques mois et il en fut de même pour la suivante. Les tirages étaient respectivement de $2000^{14}$ et de 5000 exemplaires (Biron et al., 2007, p. 123).

Cette œuvre, comme son titre le suggère, porte sur les mœurs des anciens Canadiens, à savoir les habitants de la Nouvelle-France qui ont vécu la guerre de Sept Ans et la prise du Canada par les forces britanniques. Centré sur les années ayant précédé et suivi la Conquête, l'ouvrage peut être qualifié de roman historique. L'entreprise d'Aubert de Gaspé est claire : documenter et glorifier la vie et les exploits des gens de l'époque. Sont retranscrites dans l'ouvrage la légende de la Corriveau ainsi que de nombreuses chansons populaires.

14. Hayne et Tirol affirment que le premier tirage était de 1100 exemplaires (1968b, n. p.). 
Le thème de la mémoire y est d'ailleurs central. De plus, Biron et al. affirment que "LesAnciens Canadiens a souvent été lu comme un roman de la réconciliation entre les Canadiens français et les Canadiens anglais» (ibid., p. 126). Aubert de Gaspé était très conscient de cette visée. Dans les quelques paragraphes qui servent de préface à son œuvre, il soutient en effet :

Consigner quelques épisodes du bon vieux temps, quelques souvenirs d'une jeunesse, hélas! bien éloignée : voilà toute mon ambition. Plusieurs anecdotes paraîtront, sans doute, insignifiantes et puériles à bien des lecteurs : qu'ils jettent le blâme sur quelques-uns de nos meilleurs littérateurs, qui mont prié de ne rien omettre sur les mœurs des Anciens Canadiens. (Aubert de Gaspé, 1863, p. 7)

Ainsi, lorsque le septuagénaire prend la plume pour la première fois, c'est dans le but précis et avoué de préserver les souvenirs d'une époque révolue. Il ne fait cependant aucun effort particulier pour faire entrer son œuvre dans une catégorie prédéfinie: "Que les puristes, les littérateurs émérites, choqués de ses défauts, l'appellent roman, mémoire, chronique, salmigondis, pot-pourri, peu m'importe!...», déclare-t-il (ibid., p. 8).

Selon l'abbé Casgrain, Les Anciens Canadiens a profité d'une très bonne réception critique au moment de sa publication: "Toute la presse canadienne retentit des éloges les plus flatteurs» (1871, p. 74). Le roman jouit aussi d'une large distribution; il est "en vente chez les éditeurs, MM. Desbarats, et chez tous les libraires» (Le Journal de Québec, cité dans Hayne et Tirol, 1968b, n. p.). L'abbé Casgrain lui-même ainsi que Nazaire Petit en ont publié des critiques ${ }^{15}$. Ils en vantent, entre autres, la narration, la fluidité du style ainsi que les descriptions. Le grand atout du livre est d'immortaliser «avec toutes ses traditions et ses souvenances, ses gloires et ses larmes, la plus glorieuse page de notre histoire» (Casgrain, 1871, p. 74-78). De plus, le roman est adapté en pièce de théâtre par deux professeurs du collège de l'Assomption, et la pièce y est jouée par les élèves en présence de l'auteur en 1865 (ibid., p. 87).

Les Anciens Canadiens, roman le plus connu de l'époque, a fait l'objet de trois traductions. La première, signée Georgiana M. Pennée, paraît un an après la publication de l'original, soit en 1864. Suivent

15. Casgrain présente Petit comme un jeune écrivain distingué, mais nous n'avons pas trouvé d'autres traces de l'homme. Comme Casgrain ne mentionne pas dans quel journal est parue la critique de Petit, il est difficile de poursuivre les recherches à son sujet. 
ensuite deux retraductions, dont une qui ne tarde guère. Signée par Charles G. D. Roberts, elle paraît en 1890. Il faudra ensuite attendre plus d'un siècle avant que Jane Brierley ne commette la deuxième retraduction, en 1996. Du point de vue des traductions, Les Anciens Canadiens représente un cas de figure unique, car il a été traduit et retraduit très rapidement. D'ailleurs, selon nos recherches, il s'agirait du seul roman canadien-français du XIX ${ }^{\mathrm{e}}$ siècle ayant fait l'objet d'une traduction au cours de ce même siècle et ayant été retraduit.

\subsection{La deuxième retraduction : Canadians of Old}

Penchons-nous maintenant sur la deuxième retraduction des Anciens Canadiens qui, comme mentionné ci-dessus, a été publiée en 1996 et signée par Jane Brierley. Brierley a obtenu un baccalauréat de l'Université Bishop en 1956 et une maîtrise de l'Université McGill en 1982. Son mémoire de maîtrise consistait en une traduction intégrale commentée des Mémoires d'Aubert de Gaspé, traduction qui a été publiée en 1988 sous le titre A Man of Sentiment: The Memoirs of Philippe-Joseph Aubert de Gaspé. Brierley poursuit son exploration des œuvres d'Aubert de Gaspé en traduisant ensuite Divers; intitulée Yellow-Wolf E Other Tales of the Saint Lawrence, sa traduction paraît en 1990. Les Anciens Canadiens est donc le troisième ouvrage d'Aubert de Gaspé traduit par Jane Brierley. Les trois traductions ont été publiées chez Véhicule Press, petite maison d'édition montréalaise fondée en 1973 et qui se spécialise dans la poésie, la fiction, l'essai, la traduction et l'histoire sociale (Véhicule Press, 1996-2017).

Présidente de l'Association des traducteurs et traductrices littéraires du Canada de 1990 à 1994 (ATTLC, s.d.), Jane Brierley est une traductrice littéraire reconnue dont la carrière a été jalonnée de récompenses. Sa traduction Yellow-Wolf E Other Tales of the Saint Lawrence lui a valu en 1990 le prix du Gouverneur général du Canada, décerné par le Conseil des arts du Canada. Elle a de nouveau obtenu ce prix en 2003 pour sa traduction Memoirs of a Less Travelled Road: A Historian's Life (Mémoires d'un autre siècle, Marcel Trudel) ${ }^{16}$. En 1992, elle a reçu le prix Félix-Antoine Savard pour sa traduction White Desert (Le désert blanc, Jean Éthier-Blais) ${ }^{17}$. Enfin, sa traduction The Maerlande Chronicles (Chroniques du Pays des Mères, Élisabeth

16. Plusieurs autres de ses traductions ont été en nomination pour ce même prix, dont A Man of Sentiment et Canadians of Old.

17. Le prix était remis par le Translation Center de l'Université Columbia avec la collaboration de la Délégation générale du Québec à New York (Anonyme, 1992, p. 56). 
Vonarburg) lui a valu une nomination pour le prix américain Philip K. Dick Fiction Award, ce qui est d'autant plus impressionnant que le prix avait alors toujours été décerné à des auteurs américains (Holmes, 1999, p. 122-123).

\subsection{Le projet de traduction}

Il semblerait que Jane Brierley ait elle-même pris l'initiative de produire une retraduction du roman d'Aubert de Gaspé. Dans un article publié en 1995 dans lequel elle discute de sa traduction des Anciens Canadiens et, surtout, des deux traductions précédentes, elle écrit :

In fact, knowing that I would one day want to do a new translation of this particular work, I had studiously avoided taking anything more than a brief look at the two earlier translations. (Brierley, 1995, p. 163)

I felt it was time to give Les Anciens Canadiens a fresh chance to emerge for the novel it was. With the blessing of my publisher and the Canada Council, I set to work» (ibid., p. 165).

Les propos de Brierley confirment qu'elle a bénéficié d'une subvention du Conseil des arts du Canada pour la réalisation de sa traduction. Cette information se trouve également dans la section des remerciements de la traduction : «I wish to thank the Canada Council for generously assisting this translation with a grant-in-aid» et "Published with the assistance of the Canada Council» (Aubert de Gaspé, 1996, n. p.).

Jane Brierley et son éditeur Simon Dardick ont déposé une demande dans le cadre du programme d'aide à la traduction du Conseil des arts le 22 novembre 1991 (voir Annexe I). Pour justifier le financement d'une retraduction, Dardick explique que la traduction de Charles G. D. Roberts est désormais épuisée, qu'elle regorge d'embellissements propres à l'époque victorienne et que, pour la première fois, les "Notes et éclaircissements» ${ }^{18}$ seront traduits (Simon Dardick, lettre à Katharine A. Benzekri, 22 novembre 1991; voir Annexe I). Dans un document portant la mention «Translator's Remarks» qui accompagne la demande de subvention, Jane Brierley écrit pour sa part :

Robert's English version, while more lively than the first translation, was written in a style that bordered on the pompous at times. In my view it

18. Section de plus de 50 pages représentant environ $1 / 8$ du roman, les «Notes et éclaircissements» contiennent des informations supplémentaires sur la société canadienne du XVIII e siècle. 
lacked the engaging, conversational tone of the raconteur that is one of de Gaspé's charms. (Annexe I)

La demande de financement est de l'ordre de 12303,74 \$. Selon l'estimation de la traductrice, le texte des Anciens Canadiens compte 123 037,37 mots (Annexe I). La rémunération de Brierley est donc équivalente à $0,10 \$$ le mot. Le financement sera recommandé le $1^{\text {er }}$ décembre 1991 (voir Annexe II), et Simon Dardick en recevra confirmation dans une lettre de Katharine A. Benzekri datée du 9 décembre 1991 (voir Annexe II).

La décision de retraduire Les Anciens Canadiens a été mûrement réfléchie par Brierley. Dans la préface de sa traduction, elle explique qu'une retraduction de l'ouvrage d'Aubert de Gaspé était nécessaire en raison de l'inadéquation des deux traductions précédentes. Brierley parle en connaissance de cause, puisqu'elle a procédé à une analyse comparative des traductions de Pennée et de Roberts (1995, p. 174181), analyse qu'elle a effectuée alors que sa propre traduction était au stade de la révision (ibid., p. 164). Elle présente ses résultats dans un article intitulé "Two-and-a-half Translators in Search of a Canadian of Old» (Brierley, 1995). Brierley n'a donc pas retraduit Les Anciens Canadiens dans le but premier de corriger les fautes contenues dans les deux premières traductions, mais plutôt parce qu'elle avait l'impression générale que ces traductions n'étaient pas adéquates, une impression qu'elle confirme et justifie dans son article. Brierley nourrissait aussi un intérêt marqué pour Aubert de Gaspé; après avoir traduit ses deux autres œuvres, elle souhaitait s'attaquer à l'œuvre phare de cet auteur.

Un échange de courriels avec Simon Dardick, directeur de Véhicule Press, met en lumière le fait que la retraduction des Anciens Canadiens a requis plus de temps que ce qui avait été prévu au départ. Dardick explique :

In the case of Les anciens Canadiens [sic], time-consuming research was required that touched on history and old or uncommon French terms. Jane Brierley was meticulous. As a result, the translation took longer than expected. (Communication personnelle, 13 mai 2015)

Dardick nous a fourni des lettres qu'il a échangées avec le Conseil des arts du Canada ${ }^{19}$. La première est datée du 26 octobre 1995 et signée par Dardick lui-même. On y apprend que la parution de la traduction a déjà été reportée à quelques reprises. Pour justifier le fait que le projet

19. Le contenu de ces lettres est reproduit avec la permission de Simon Dardick et de Véhicule Press. Nous tenons à les remercier pour leur précieuse contribution. 
de traduction a pris plus de temps que prévu, Dardick écrit :

For the first time, de Gaspés annotations and notes to the original text will be included in an English edition. Jane Brierley's research has been meticulous and the resulting translation is truly a remarkable achievement. (Simon Dardick, lettre à Carole Boucher, 26 octobre 1995)

Dardick ajoute que la traduction sera imprimée sous peu. Il reçoit une réponse de Carole Boucher, responsable des programmes de la section des lettres et de l'édition du Conseil des arts, le 19 décembre 1995, qui lui confirme que le deuxième versement de la subvention, un montant de 6000 \$, parviendra sous peu à la maison d'édition et qu'il restera 304 \$ à verser à la parution de la traduction (Carole Boucher, lettre à Simon Dardick, 19 décembre 1995). Puis, en 1996, Dardick reçoit une lettre datée du $1^{\text {er }}$ avril dans laquelle on peut lire que Véhicule Press a reçu le 9 décembre 1991 une subvention de 12304 \$ de la part du Conseil des arts du Canada pour la traduction des Anciens Canadiens. Dans cette même lettre, on demande à Véhicule Press de fournir un exemplaire de la traduction avant le 30 avril 1996. On ajoute que si le Conseil des arts ne reçoit pas la traduction pour cette date, le dossier sera fermé et le dernier versement sera annulé, sans compter que Véhicule Press devra rembourser l'argent déjà reçu (Marcel Hull, lettre à Simon Dardick, $1^{\text {er }}$ avril 1996). Cette dernière correspondance est particulièrement parlante. Il s'est écoulé plus de cinq ans entre la demande de subvention et la publication de la traduction, à la fin de l'année 1996. Finalement, une lettre de Carole Boucher datée du $1^{\text {er }}$ avril 1997 et obtenue dans le cadre de la Loi sur l'accès à l'information nous apprend que le Conseil des arts a reçu un exemplaire de Canadians of Old.

\subsection{Réception de la traduction dans le Canada anglophone}

D'entrée de jeu, soulignons que le projet de traduction des Anciens Canadiens par Jane Brierley n'est pas passé inaperçu. Déjà, en 1992, lorsque le quotidien Le Soleil souligne la nomination de Brierley pour le prix Félix-Antoine Savard, il est annoncé qu'elle «travaille actuellement à la traduction du célèbre roman historique d'Aubert de Gaspé, Les Anciens Canadiens» (Le Soleil,1992,p. C3). Nous avons aussi trouvé quatre articles datant de 1997 qui mentionnent la nomination de Brierley pour le prix du Gouverneur général dans la catégorie traduction du français vers l'anglais. Ces articles ont été publiés dans The Canadian Press NerwsWire (22 octobre 1997), The Globe and Mail (23 octobre 1997), le Edmonton Journal (23 octobre 1997) et le Daily 
Nerws (23 octobre 1997). Nous avons de plus découvert une annonce de parution dans le Globe and Mail du $1^{\text {er }}$ mars 1997. Cette dernière met de l'avant la traduction et la traductrice : l'auteure explique que le roman a déjà fait l'objet de traductions, mais que «Brierley wants to improve on ornately wordy early translations [sic], and on the Charles G. D. Roberts version (1890), which while readable, "took an adaptive approach that, in my view, went too far"» (Kirchoff, 1997, p. D15).

Deux critiques de la traduction ont par ailleurs été publiées. La première, parue dans The Gazette le 5 janvier 1997, affirme que la traduction des œuvres d'Aubert de Gaspé pourrait contribuer à la réconciliation entre francophones et anglophones (Kozinska, 1997, p. C5). On y trouve également relayés les propos d'une entrevue réalisée avec Brierley qui permettent d'apprendre que, selon la traductrice, la valeur des Anciens Canadiens ne repose pas seulement sur son caractère historique, mais également sur son caractère social :

Not to knock the historical context, I think the importance of the book lies in the social aspect, and by that I include personal relationships and the sense of fun that people have. They used to have an awfully good time, and this comes through. (Brierley citée dans Kozinska, 1997, p. C5)

L'article nous informe sur la vision que Brierley a d'Aubert de Gaspé, de ses œuvres et de la littérature de l'époque, mais il n'est toutefois pas question de la traduction en tant que telle. La deuxième critique paraît dans la revue savante University of Toronto Quarterly et est signée par la traductologue Jane Koustas (1998/1999, p. 329). Pour Koustas, il ne fait aucun doute qu'une retraduction des Anciens Canadiens était nécessaire :

"One only has to read the caption of the illustration on the first page of Penné's [sic] translation, "How are you Jose? and how have you left them all at home?" and the title of Roberts's final version [Cameron of Lochiel, NDA] to appreciate the need for a makeover.» (ibid., p. 340)

Brierley fait plus que produire une bonne traduction, elle apporte son expérience en tant qu'experte des œuvres d'Aubert de Gaspé, toutes ayant demandé beaucoup de recherches. Koustas écrit également: "Canadians of Old [...] offers English readers the first complete and delightfully readable version of Aubert de Gaspe's classic novel» (ibid.), apposant ainsi un jugement favorable à la traduction de Brierley.

Finalement, Brian Busby, écrivain et historien de la littérature, écrit dans son blogue que la traduction de Brierley est supérieure à celle de Roberts : 
I imagine that Roberts' translation is the most read (the NCL edition sold nearly 1800 copies in the first six months alone); a great shame since it has been surpassed by Jane Brierley's 1996 translation. The only one currently in print, it is highly recommended [...]. (24 septembre 2009, n. p.).

Bref, la traduction de Brierley est le fruit d'une initiative personnelle et de plusieurs années de travail. Brierley avait déjà traduit les deux autres ouvrages d'Aubert de Gaspé et voulait boucler la boucle en traduisant toutes ses œuvres. Elle effectuera d'ailleurs des recherches méticuleuses afin de trouver des équivalents aux termes canadiensfrançais. Malgré tout, la traduction de Brierley a été moins remarquée que celle de Charles G. D. Roberts.

\section{Conclusion}

Les traductions que nous avons examinées dans cet article ont été produites dans des contextes différents, mais il va sans dire qu'elles ont contribué à la diffusion des écrits canadiens-français du XIX ${ }^{\mathrm{e}}$ siècle dans l'Amérique du Nord anglophone. Elles ont été effectuées après l'adoption de la Loi sur les langues officielles (1969) et la mise en place du programme d'aide à la traduction du Conseil des arts du Canada (1972), dans un contexte favorable aux échanges entre les deux groupes linguistiques majoritaires du Canada. De plus, elles ont été financées par divers organismes subventionnaires. Les Presses de l'Université de Toronto ont ainsi bénéficié en 1974 du soutien financier du Conseil des arts de l'Ontario et de la fondation McLean pour la publication de la version anglaise d'Angéline de Montbrun. Les subventions reçues visaient à minimiser les coûts associés à la publication; rien n'indique que le traducteur ait été rémunéré à même ce financement. Les maisons d'édition montréalaises Robert Davies Publishing et Véhicule Press ont pour leur part bénéficié du soutien du Conseil des arts du Canada pour publier respectivement The Influence of a Book en 1993 et Canadians of Old en 1996.

Il n'est sans doute pas anodin que les deux traductions produites dans les années 1990 aient été soutenues par le Conseil des arts du Canada, contrairement à celle produite au début des années 1970. En effet, au moment de la publication d'Angéline de Montbrun en version anglaise, le programme d'aide à la traduction du Conseil des arts n'existait que depuis deux ans. On peut penser qu'il était encore peu connu, surtout en dehors du Québec et du cercle des traducteurs littéraires. Il est possible également qu'Yves Brunelle ait entrepris sa 
traduction avant même la mise sur pied du programme. À l'inverse, le programme était bien établi au début des années 1990, et on peut penser que les traductrices et les éditeurs qui sont intervenus dans la publication de The Influence of a Book et de Canadians of Old le connaissaient bien, notamment parce qu'ils évoluaient dans le milieu québécois de la traduction littéraire.

Cela dit, les traductions en question sont le résultat d'initiatives personnelles, à savoir d'Yves Brunelle, grand défenseur de la littérature québécoise, de Robert Davies, éditeur spécialisé dans les ouvrages québécois anciens, et de la traductrice littéraire Jane Brierley, admiratrice des œuvres de Philippe Aubert de Gaspé. On ne saurait voir dans leur publication un désir institutionnel de promouvoir la traduction des œuvres du XIX ${ }^{\mathrm{e}}$ siècle (et ce, tant vers le français que vers l'anglais). Il n'en demeure pas moins qu'en réduisant le risque financier des éditeurs, les organismes subventionnaires permettent la diffusion de la littérature canadienne-française du XIX ${ }^{\mathrm{e}}$ siècle au-delà de ses frontières. De plus, comme toutes les traductions à l'étude ont bénéficié d'un appui financier, nous sommes d'avis qu'il y a une corrélation entre obtention d'une subvention et production d'une traduction. Nous n'irons toutefois pas jusqu'à affirmer que, sans ce soutien, les romans seraient demeurés non traduits: les projets de traduction ont été menés par des personnes engagées, qui auraient probablement trouvé des solutions créatives pour les réaliser. Il est néanmoins évident que les organismes subventionnaires facilitent et accélèrent la réalisation de tels projets et permettent le rayonnement des auteurs canadiensfrançais les plus connus.

\section{Références}

Abrams, M. H. et Geoffroy Galt Harpham (2015). A Glossary of Literary Terms. Stamford, Centage Learning.

Anonyme (1992). «Prix et distinctions». Lettres québécoises : la revue de l'actualité littéraire, 67, p. 56.

Association des traducteurs et traductrices littéraires du Canada (AATLC) (s.d.). «Brierley, Jane». [https://www.attlc-ltac.org/fr/lequipe/].

Aubert de Gaspé, Philippe (fils) (1837). L'Influence d'un livre. Roman historique. Québec, William Cowan et fils.

Aubert de Gaspé, Philippe (fils) (1864). «Le chercheur de trésors ou L'Influence d'un livre». In Direction du foyer canadien, dir. La littérature canadienne de 1850 à 1860. Tome II, Québec, G. et G. E. Desbarats. La Bibliothèque électronique du Québec, p. 123-220.

Aubert de Gaspé, Philippe (fils) (1993). The Influence of a Book. Trad. Claire Holden Rothman. Montréal, Robert Davies Publisher. 
Aubert de Gaspé, Philippe (père) (1863). Les Anciens Canadiens. Québec, Desbarats et Derbishire.

Aubert de Gaspé, Philippe (père) (1864). The Canadians of Old. Trad. Georgiana M. Pennée. Québec, G. et G. E. Desbarats.

Aubert de Gaspé, Philippe (père) (1890). The Canadians of Old. Trad. Charles G. D. Roberts. New York, D. Appleton and Company.

Aubert de Gaspé, Philippe (père) (1996). The Canadians of Old. Trad. Jane Brierley. Montréal, Véhicule Press.

Biron, Michel, François Dumont et Élisabeth Nardout-Lafarge (2007). Histoire de la littérature québécoise. Montréal, Boréal.

Boivin, Aurélien (2006). «Le roman du terroir». Québec français, 143, p. 32-37. Boucher, Carole (1995). Lettre à Simon Dardick, 19 décembre 1995.

Brierley, Jane (1995). «Two-and-a-half Translators in Search of a Canadian of Old». In S. Simon, dir. Culture in Transit: Translating the Literature of Quebec. Montréal, Véhicule Press, p. 163-184.

Brooks, Richard A. (1980). A Critical Bibliography of French Literature. Volume 6: The Twentieth Century. Part 1: General Subjects and Principally the Novel before 1940. Syracuse, Syracuse University Press.

Brunet, Manon (1997). «Henri-Raymond Casgrain et la paternité d'une littérature nationale ». Voix et Images, 22,2, pp. 205-224.

Brunet, Manon (2005). «Angers, Félicité dite Laure Conan». In Dictionnaire biographique du Canada. Université Laval/Université de Toronto. [http:// www.biographi.ca/fr/bio/angers_felicite_15F.html].

Busby, Brian (24 septembre 2009). «Old Folks». The Dusty Bookcase. A Journey through Canada's Forgotten, Neglected and Suppressed Writing [blogue]. [http://brianbusby.blogspot.com/2009/09/old-folks.html].

Carr, Thomas M. (1998). «Consolation and the Work of Mourning in Angéline de Montbrun». The French Review, 71, 6, p. 997-1006.

Casgrain, Henri-Raymond (1871). Philippe Aubert de Gaspé. Québec, Atelier typographique de Léger Brousseau.

Cimon, Anne (1994). «The Philosopher's Stone». Matrix, 43, p. 76-77.

Conan, Laure (1884). Angéline de Montbrun. Québec, Imprimerie Léger Brousseau.

Conan, Laure (1891). À l’ouvre et à l'épreuve. Québec, C. Darveau.

Conan, Laure (1909). The Master Motive: A Tale of the Days of Champlain, Trad. Theresa A. Gethin. St-Louis (MI), B. Herder.

Conan, Laure (1974). Angéline de Montbrun. Trad. Yves Brunelle. Toronto, Presses de l'Université de Toronto.

Conseil des arts du Canada (n.d.). «L'art au coeur de nos vies». Conseil des arts $d u$ Canada. [https://conseildesarts.ca/].

Dardick, Simon (1991). Lettre à Katharine A. Benzekri, 22 novembre 1991.

Dardick, Simon (1995). Lettre à Carole Boucher, Re: Translation of Les Anciens Canadiens by Philippe Aubert de Gaspé, 26 octobre 1995.

Delisle,Jean (1987). La traduction au Canada/Translation in Canada, 1534-1984. 
Ottawa, Les Presses de l'Université d'Ottawa.

Delisle, Jean (2001). "Canadian Tradition». In M. Baker et G. Saldanha, dir. The Routledge Encyclopedia of Translation Studies. Londres et New York, Routledge, p. 356-363.

Galarneau, Claude (1986). «Autrefois, le commerce du livre». Cap-auxDiamants, 2, 1, p. 3-7.

Gosselin, Line (2005). «Marchand, Joséphine (Dandurand)». In Dictionnaire biographique du Canada. Université Laval/Université de Toronto. [http:// www.biographi.ca/fr/bio/marchand_josephine_15F.html].

Grignon, Claude Henri (1978). The Woman and the Miser. Trad. Yves Brunelle. Eugene (OR), Harvest House.

Grutman, Rainier et Claude La Charité (2013). Philippe Aubert de Gaspé père et fils en revue. Québec, Presses de l'Université du Québec et Revue voix et image, coll. «De vives voix».

Hayne, David M. (1986). «Institution québécoise et institution française au $\mathrm{XIX}^{\mathrm{e}}$ siècle». In M. Lemire, dir. L'institution littéraire. Université Laval, Centre de recherche en littérature québécoise, p. 51-60.

Hayne, David M. (2009). «Compte rendu de Relire Angéline de Montbrun au tournant du siècle. E.D. Blodgett et Claudine Potvin, dir. Québec, Nota Bene, 2006, 458 p.» University of Toronto Quaterly, 78, 1, p. 618-620.

Hayne, David M. et Marcel Tirol (1968a). «Aubert de Gaspé, Philippe-IgnaceFrançois». In Bibliographie critique du roman canadien-français, 1837-1900. Toronto, Les Presses de l'Université de Toronto, n. p.

Hayne, David M. et Marcel Tirol (1968b). «Aubert de Gaspé, PhilippeJoseph». In Bibliographie critique du roman canadien-français, 1837-1900. Toronto, Les Presses de l'Université de Toronto, n. p.

Hayne, David M. et Marcel Tirol (1968b). Bibliographie critique du roman canadien-français, 1837-1900. Toronto, Les Presses de l'Université de Toronto, n. p.

Hillinger, Alexandra (2017). La production et la réception dans l'Amérique du Nord anglophone des traductions de langue anglaise de romans canadiens-français publiés au XIX $X^{e}$ siècle. Thèse de doctorat. Université Concordia. [https:// spectrum.library.concordia.ca/982627/1/Hillinger_PhD_F2017.pdf].

Holmes, Gillian (1999). Chatelaine Presents Who's Who of Canadian Women. Webcom Limited.

Hull, Marcel (1996). Lettre à Simon Dardick, Re: file 6035-91-0375, $1^{\text {er }}$ avril 1996.

Kirchoff, H. J. (1997). «Back in Paper A World of My Own: A Dream Diary». The Globe and Mail, Toronto, $1^{\text {er }}$ mars.

Kozinska, Dorota (1997). «Voyage into History: Intimate Side of $19^{\text {th }}$ Century Quebec Life Captured in Work of Philippe-Joseph Aubert». The Gazette, Montréal, 5 janvier.

Koustas, Jane (1998/99). «Translations ». University of Toronto Quarterly, 68, 1, p. 328-344. 
Lacourcière, Luc (n. d.). «Aubert de Gaspé, Philippe-Joseph ». In Dictionnaire biographique du Canada. Université Laval/Université de Toronto. [http:// www.biographi.ca/fr/bio/aubert_de_gaspe_philippe_joseph_10F.html].

Lambton, John (Earl of Durham) (1839). Report on the Affairs of British North America. Toronto, Printed by Robert Stanton.

Lareau, Edmond (1874). Histoire de la littérature canadienne. Montréal, John Lovell.

Le Soleil (1992). «Le prix F.-A.-Savard remis à Jane Brierley». Québec, $1^{\mathrm{er}}$ juin. Lemire, Maurice (1980). Dictionnaire des cuvres littéraires du Québec: 19001939. Tome II. Montréal, Fides.

Lemire, Maurice (1994). «Les revues littéraires au Québec comme réseaux d'écrivains et instance de consécration littéraire (1840-1870)». Revue d'histoire de l'Amérique française, 47, 4, p. 521-550.

Linteau, Paul-André, René Durocher et Jean-Claude Robert (1979). Histoire du Québec contemporain. Montréal, Boréal express.

Milner, Philip (1997). «The Complicated Code, Town and Gown: It Made Perfect Sense to Saints, Politicians, and Eastern Nova Scotia Catholics». The Daily News, Halifax, 23 janvier.

Noreau, Michel A. (2010). De Gaspé fils 1814-1841: La vie tumultueuse du premier romancier québécois. Québec, Auto-édition.

Open Book Toronto (2009). «Ten Questions with Claire Holden Rothman». Open Book Toronto. [http://staff.openbooktoronto.com/news/ten questions_with_claire_holden_rothman].

Perkes,Carolyn (1997). «The Québécois and English Canadian Literary System: Translation, Republishing, and the Preface». In S. Tötösy de Zepetnek et I. Sywenky, dir. The Systemic and Empirical Approach to Literature and Culture as Theory and Application. Edmonton, Université de l'Alberta, p. 309-320.

Robert, Lucie (1989). L'institution du littéraire au Québec. Sainte-Foy, Les Presses de l'Université Laval.

Stratford, Philip (1977). «Literary Translation in Canada: A Survey». Meta, 22, 1, p. 37-44.

Thatcher, Wendy (1994). «Quebec's First Novel is Dark, Bloody Affair». The Gazette, Montréal, 2 avril.

Woodsworth, Judith (2001 [1998]). «History of Translation». In M. Baker, dir. Routledge Encyclopedia of Translation Studies, Londres et New York, Routledge. [Version numérique], n. p.

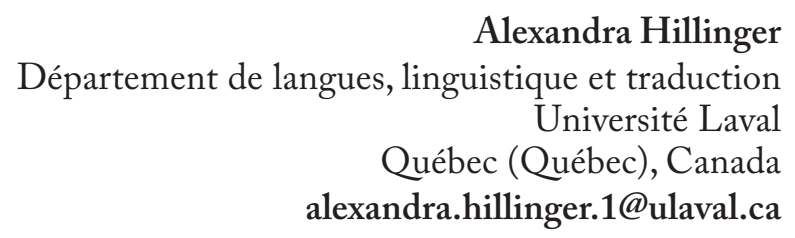




\section{Annexe I}

\section{Demande de financement pour la traduction des Anciens Canadiens dans le cadre du programme d'aide à la traduction du Conseil des arts du Canada}

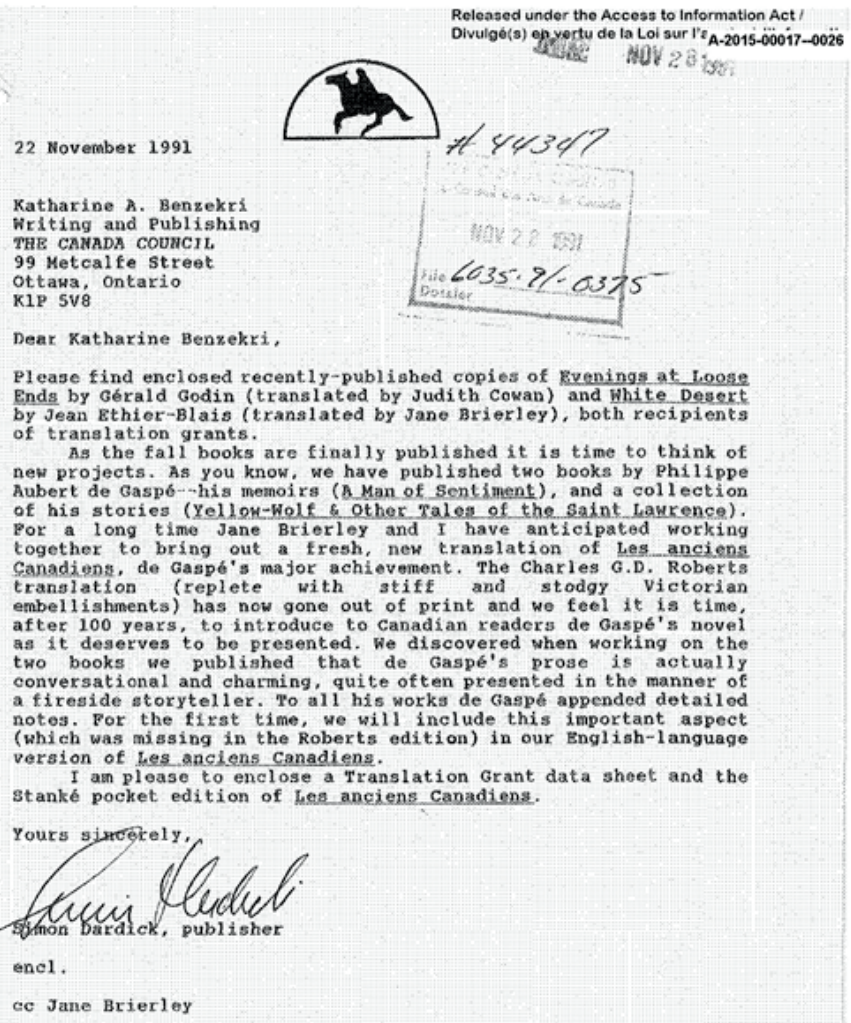

VÉHICULE PRESS • P.O.B. 12S • PLACE DU PARC STATION • MONTRÉAL, QUÉBEC • H2W 2M99 (514) 844.6073 


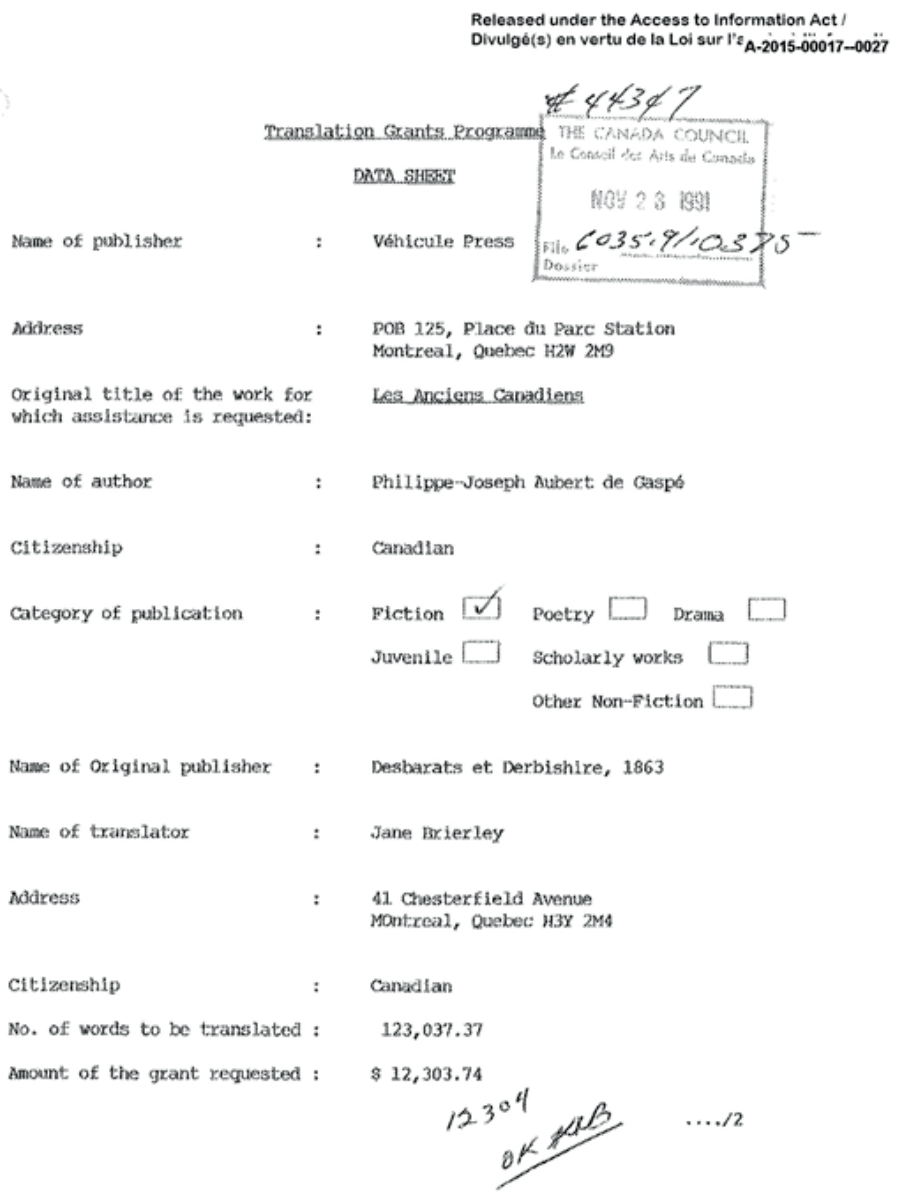


Do you presently hold translation rights for this title?

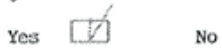

If so, please speclfy terias :

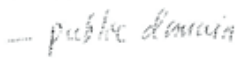

If not, state present status of negotiations:

To publish the present work in its new language edition, do you intend:

a) to use a portion of your Council Block grant?

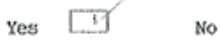

b) to subuit an application to the councll for a project grant?

or

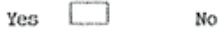

c) to submit an application to the Social Sciences Federation of canada for a publication grant?

Translator's signature:

Publisher's slgnature:

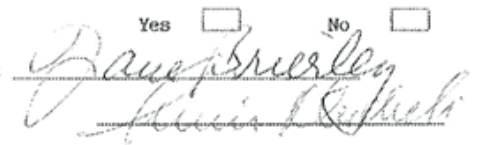

When applying under the Council's programe of ald to Translation, it is essentlal that the publisher submits one or two coples of the original. vork along with a sample translation of at least twenty-five (25) pages. In certain clrcumstances, the Councll may require additional Information. 
Released under the Access to Information Act I Divulgé(s) en vertu de la Loi sur I'z A-2015-00017-0029

Word Count Calculation for Translation Proposal, Les Anciens Canadjens (1863) by Philippe--Joseph Aubert de Gaspé

(Por this purpose, a copy of the pocket edition (stanke 1997) has been used. This is a reduced page reproduction of the Bcacheain 1913 edition. the actual trasslation vill be based on the fitst edition (onebec: Desharats et Derbishize, 1063) vith errate noted by the author.]

\section{A. MAIN TEXX' (pD. $11-242$ )}

Sample to establish average word par page: page 215, with 12 paragraphs page 219 , w1th 3 paragraphs Average: $398+435 \div 2=$

$398 \quad$ words $485 \quad$ words 441.5 wds per page

No of whole pages (excl. blank page on 149): 1/4 pages: $(63,102,127,148,180,227,242)$ $1 / 2$ pages: $(17,82,95,137,165)$ $3 / 4$ pages: (1.8 chapter headings \& po. 27,112, Total pages of main text to be counted: 199 pages 1.75 pages 2.5 pages 20 pages 223.25 pages

Subtotal words: $(441.5 \times 223.25)$

98564.875 words

\section{B. AUTHOR'S "NOMES ET ECT.NYRCTSSIPEANTS"}

(an integral part of the original book, pp. $243-298$. This section has smaller type, less space between lines.)

Sample to establish average word per page: page 249, with 8 paragraphs page 248 , with 4 paragraphs Average words pex page $(420+584 \div 2)$

420

No. of whole pages:

$1 / 4$ pages: $(244,273,280,282,284)$

$1 / 2$ pages: $(277,290,294)$

$3 / 4$ pages: (251, 259, 279, 297)

43 pages

1.25 page

.5 pages

pages

Total pages of "Notes et Éclaixclssentents": 48.75 pages

Subtotal words: $(48.75 \times 502)=$

$24472.5 \quad$ words

***

TOTAL, WORns: $\quad(98564.875+24472.5)$

$123,037.37 \quad$ words

TORAL TRANSLATION GRANT-IN-AID APPLICATION:

$\$ 12303.74$ 


\title{
Le soutien accordé par les organismes subventionnaires
}

Released under the Access to Information Act ? Divulgé(s) en vertu de la Loi sur l'a A-2015-00017-0030

Translator's. Bemarks

\author{
by enilippe-Jomeph Aubext de Gaspé \\ (1786-1871)
}

les Anciens Canadiens vas originally published in 1863, and has remalned in print in various editions. Fides brought cut a mass paperback copy in 1979, and Stanké published a wass paperback edition in 1987 (a reduced-pagé facsimile of the Beauchemin edition of 1913, which had unigue decorative features).

The first English translation appeared in 1864 (trans, Georgland M. Pennoe, Quebec: G. \& G.B. Desbarats), a rather pedestrian translation that appeared in a slightly revised form as cameron of Lochedil. Both these editions are out of print.

In 1890 an English translation by Charles G.D. Roberts was brought out: by D. Appleton and Conpany, and this version has since been republished by Mcclelland \& Stewart (1974) in thelx Ner Caradian Library serles, no. 106. The "Notes et sclaircissenents" section, an important feature of Les Anciens cavadiens that is almost a small historical meinolr in 1tself, was included in this edition, but in French only. A phone call to the publisher on 21 Novenber 1991 confirmed that this edition is no longer on the publisher's list, and is out of print.

Roberts' English version, whlle wore lively than the flrst translation, was written in a style that bordered on the pompous at times. In my viev it lacked the engaging, conversational tone of the zaconteux that is one of de Gaspe's great: charias.

I prepared the first English translations of de Gaspe's two other publisthed vorks, and these versions have met with considerable critical success." Readers have expressed thelx enjoyment of the books, as well as thelr appreciation of the translator's additional explanatory material, which provides helpful background for works written more than a century ago.

I feel that it is not only appropriate, but necessary that de Gasps's maln work appear in a bew Bnglish version that does fustice to his great talent and malntalns the stylistic perspective established in A Man of Sentiment and Yellow Nolf. All his published books would then be avallable to the English reading public.

Jane Brierley

* The English version of de Gaspé's Mrpoires (A Man of Sentiment (Montreal: vehicule Press, 1988) was shortlisted for the Governor General' is Bnglish translation award. The translation of Divers ( $\mathrm{X}_{e}$ llow nolf \& other Tales of the saint Lawrence (Montreal: vbhicule Press, 1990) won the Governor General's avard. 


\section{Annexe II}

Octroi d'une subvention pour la traduction des Anciens Canadiens dans le cadre du programme d'aide à la traduction du Conseil des arts du Canada

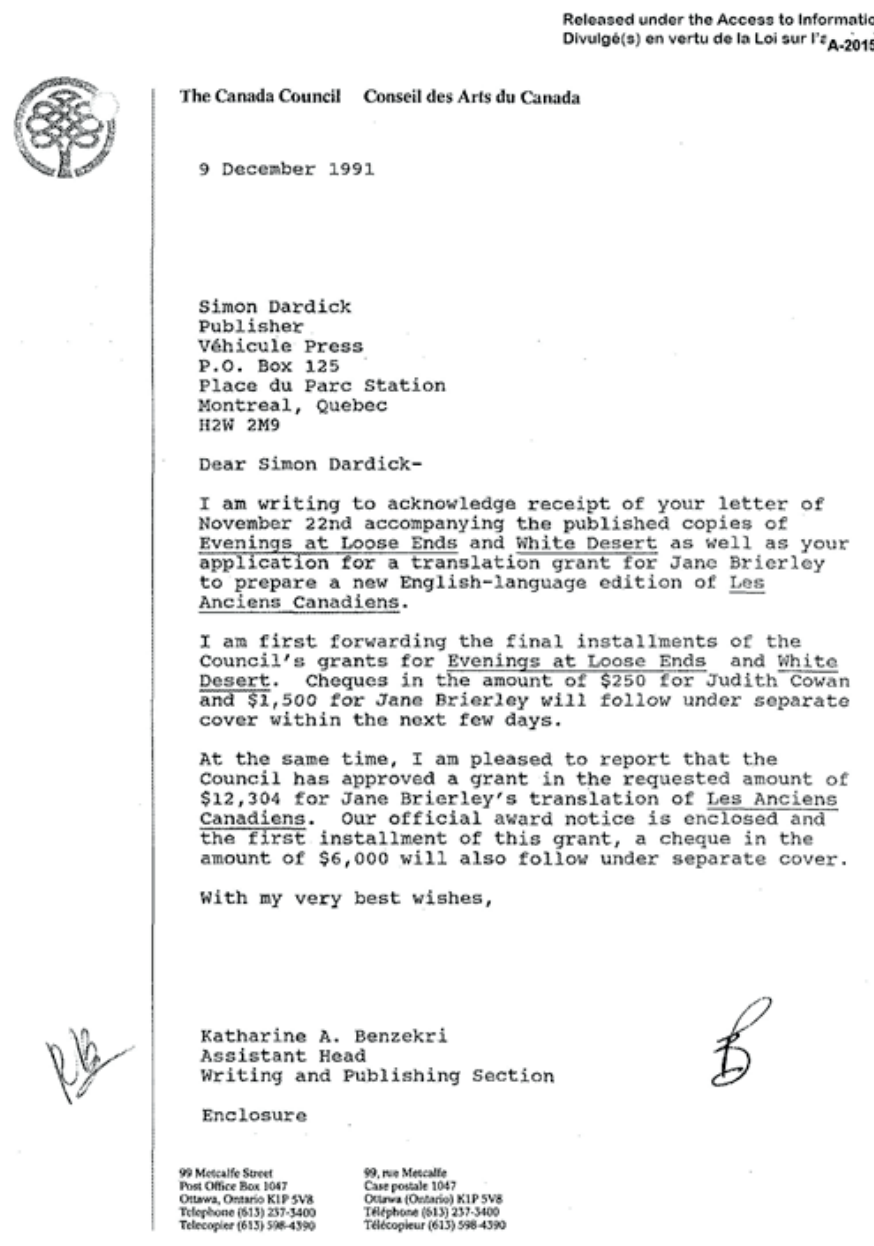




\begin{tabular}{|c|c|}
\hline & Recommendation for Director's Grant \\
\hline Section: & Writing and Publishing \\
\hline Program: & Translation Grants \\
\hline Applicant: & $\begin{array}{l}\text { Vehicule Press, Montreal, Quebec } \\
\text { Simon Dardick, Publisher }\end{array}$ \\
\hline Title: & Les Anciens Canadiens \\
\hline Author: & Philippe-Joseph Aubert de Gaspe \\
\hline Category: & Fiction \\
\hline Translator: & Jane Brierley \\
\hline Application date: & $28-11-91$ \\
\hline Recommendation date: & $01-12-91$ \\
\hline Amount requested: & $\$ 12,304$ \\
\hline Amount recommended: & $\$ 12,304$ \\
\hline Purpose: & Translation grant \\
\hline Method of assessment: & Assessor/Council Officer \\
\hline
\end{tabular}

\section{Comments}

There have been two previous translations - both very dated (1864 and 1890) and out of print. The publisher already has de Gaspé's Mómoire and Divers in Erglish editions and the completion of this project will mean that all his published books are available in English. The translator chosen translated the other de Gaspe titles and was shortlisted in 1988 for the Mémoires and won the 1990 G.G. Prize for translation for Divers. We have in the past supported new translations when, as is the case, the initial ones were dated and out of print Alan Brown's new translation of Maria Chapdelaine for instance.

Financial Analysis

Very judicious calculation of the wordcount prepared by translator - on file.

\section{Recommendation}

1 recommend therefore a grant in the amount of $\$ 12,304$.
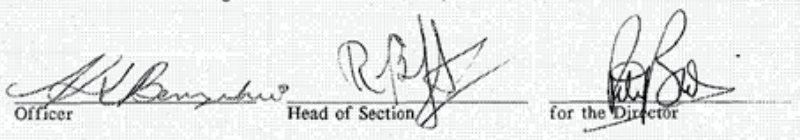

File number:

6035-91-0375 